\title{
Existence of Entire Solutions for Quasilinear Elliptic Systems under Keller-Osserman Condition
}

\author{
YUAN ZHANG AND ZUODONG YANG 1 \\ Institute of Mathematics, School of Mathematical Sciences, \\ Nanjing Normal University, \\ Jiangsu Nanjing 210023, China. \\ zdyang-jin@263. net
}

\begin{abstract}
In this paper, we study the existence of entire solutions for the following elliptic system

$$
\triangle_{\mathrm{m}} \mathrm{u}=\mathrm{p}(\mathrm{x}) \mathrm{f}(v), \triangle_{\mathrm{l}} v=\mathrm{q}(x) \mathrm{g}(\mathrm{u}), x \in \mathbf{R}^{\mathrm{N}},
$$

where $1<m, l<\infty, f, g$ are continuous and non-decreasing on $[0, \infty)$, satisfy $f(t)>$ $0, g(t)>0$ for all $t>0$ and the Keller-Osserman condition. We establish conditions on $p$ and $q$ that are necessary for the existence of positive solutions, bounded and unbounded, of the given equation.
\end{abstract}

\section{RESUMEN}

En este artículo estudiamos la existencia de soluciones enteras para el siguiente sistema elíptico

$$
\triangle_{\mathrm{m}} \mathrm{u}=\mathrm{p}(x) \mathrm{f}(v), \triangle_{\mathrm{l}} v=\mathrm{q}(x) \mathrm{g}(\mathrm{u}), x \in \mathbf{R}^{\mathrm{N}},
$$

donde $1<\mathrm{m}, \mathrm{l}<\infty$, f,g son continuas y no-decrecientes en $[0, \infty)$, satisfaciendo $f(t)>0, g(t)>0$ para todo $t>0$ y la condición de Keller-Osserman. Establecemos condiciones sobre $p$ y qy que son necesarias para la existencia de soluciones positivas, acotadas y no acotadas de la ecuación dada.

Keywords and Phrases: quasi-linear elliptic system; sub/super-solution; large solution; existence.

2010 AMS Mathematics Subject Classification: 35J50; 35J57; 35J62; 35J92.

\footnotetext{
${ }^{1}$ Project Supported by the National Natural Science Foundation of China(No.11171092); the Natural Science Foundation of the Jiangsu Higher Education Institutions of China(No.08KJB110005)
} 


\section{Introduction}

In this paper, we investigate the following quasilinear elliptic system

$$
\left\{\begin{array}{l}
\triangle_{\mathrm{m}} u=p(x) f(v), \quad x \in \mathbf{R}^{N}, \\
\triangle_{\mathfrak{l}} v=q(x) g(u), \quad x \in \mathbf{R}^{N} .
\end{array}\right.
$$

where $1<\mathrm{m}, \mathrm{l}<\infty, \mathrm{N} \geq \max \{\mathrm{m}, \mathrm{l}\}+1, \triangle_{\mathrm{m}} \cdot=\operatorname{div}\left(|\nabla \cdot|^{\mathrm{m}-2} \nabla \cdot\right)$. Denote $\mathrm{d}=\min \{\mathrm{m}, \mathrm{l}\}$, and see that $d>1$. By an entire large solution $(u, v)$, we mean a pair of functions $u, v \in C^{1}\left(\mathbf{R}^{N}\right)$ that satisfies (1.1) at every point of $\mathbf{R}^{\mathrm{N}}$ and

$$
\lim _{|x| \rightarrow \infty} u(x)=\lim _{|x| \rightarrow \infty} v(x)=\infty .
$$

First, we introduce the assumptions below:

$(\mathrm{H} 1) \mathrm{p}, \mathrm{q}: \mathbf{R}^{\mathrm{N}} \rightarrow[0, \infty)$ and $\mathrm{f}, \mathrm{g}:[0, \infty) \rightarrow[0, \infty)$ are continuous and nontrival functions;

(H2) $f$ and $g$ are nondecreasing on $[0, \infty)$ and $f(t)>0, g(t)>0$ for all $t>0$;

(H3) $\mathrm{H}(\infty)=\lim _{\mathrm{r} \rightarrow \infty} \mathrm{H}(\mathrm{r})=\infty$, where

$$
H(r)=\int_{c}^{r} \frac{d t}{\sqrt[d]{F(t)+G(t)}}, r \geq c>0 ; \quad F(t)=\int_{0}^{t} f(s) d s, G(t)=\int_{0}^{t} g(s) d s .
$$

and $c$ is a positive constant. Notice that $H^{\prime}(r)=\frac{1}{\sqrt[d]{\mathrm{F}(r)+G(r)}}>0, \forall r>c$, so $H$ has the inverse function $\mathrm{H}^{-1}$ on $[0, \infty)$. Denote

$$
\begin{aligned}
& \phi_{1}(r):=\max _{|x|=r} p(x), \quad \phi_{2}(r):=\min _{|x|=r} p(x), \\
& \psi_{1}(r):=\max _{|x|=r} q(x), \quad \psi_{2}(r):=\min _{|x|=r} q(x) .
\end{aligned}
$$

Since 1980s, many important results have been obtained for quasilinear elliptic systems. We will introduce some results in the following. Existence and non-existence of solutions of the quasilinear elliptic system

$$
\left\{\begin{array}{l}
\operatorname{div}\left(|\nabla u|^{m-2} \nabla u\right)+f(u, v)=0, \quad x \in \mathbf{R}^{\mathbf{N}} \\
\operatorname{div}\left(|\nabla v|^{l-2} \nabla v\right)+g(u, v)=0, \quad x \in \mathbf{R}^{\mathbf{N}}
\end{array}\right.
$$

has gained much attention recently. See, for example, $[3,4,10,15,19,21,22]$.

When $p=q=2$, system (1.3) becomes

$$
\begin{cases}\triangle u+f(u, v)=0, & x \in \mathbf{R}^{\mathbf{N}} \\ \triangle v+g(u, v)=0, & x \in \mathbf{R}^{\mathbf{N}}\end{cases}
$$

for which the existence and the non-existence of positive solutions and positive boundary blow-up solutions have been investigated extensively. We list here, for example, [1,2,5,6,12-14,16] and refer to the references therein. 
When $p=q=2, f=-a(|x|) v^{\alpha}, g=-b(|x|) u^{\beta}$, system (1.3) becomes

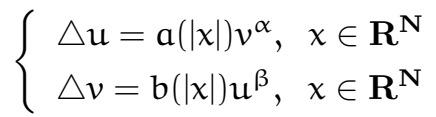

for which existence results for positive boundary blow-up solutions can be found in a recent paper by Lair and Wood [12]. Lair and Wood established that all positive entire radial solutions of (1.4) are boundary blow-up provided that

$$
\int_{0}^{\infty} \operatorname{ta}(t) d t=\infty, \quad \int_{0}^{\infty} t b(t) d t=\infty
$$

If, on the other hand

$$
\int_{0}^{\infty} \operatorname{ta}(\mathrm{t}) \mathrm{dt}<\infty, \quad \int_{0}^{\infty} \mathrm{tb}(\mathrm{t})<\infty,
$$

then all positive entire radial solutions of (1.4) are bounded.

F. Cirstea and V.D. Radulescu [1], extended the above results to a larger class of systems

$$
\left\{\begin{array}{l}
\triangle \mathrm{u}=\mathrm{a}(|x|) \mathrm{g}(v), \quad x \in \mathbf{R}^{\mathbf{N}} \\
\triangle v=\mathrm{b}(|x|) \mathrm{f}(\mathrm{u}), \quad x \in \mathbf{R}^{\mathbf{N}}
\end{array}\right.
$$

In recent years, Zhijun Zhang et al.[23] studied the following semilinear elliptic systems

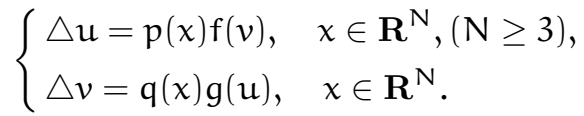

They obtained the existence and nonexistence of solutions for (1.5) by considering a set of hypotheses on $p, q, f$ and $g$.

Z.D. Yang [19], extended the above results to a class of systems

$$
\left\{\begin{array}{l}
\operatorname{div}\left(|\nabla u|^{m-2} \nabla u\right)=a(|x|) g(v), \quad x \in \mathbf{R}^{\mathbf{N}}, \\
\operatorname{div}\left(|\nabla v|^{l-2} \nabla v\right)=b(|x|) f(u), \quad x \in \mathbf{R}^{\mathbf{N}} .
\end{array}\right.
$$

Motivated by the results of the papers [19-23]. In this paper, we consider the quasilinear elliptic system (1.1). We modify the method developed by Zhang et al.[23] and extend partial results of [23] to a quasilinear elliptic system (1.1).

\section{Main Results}

In order to establish our main result, we introduce the following hypotheses :

(H4) $r^{N-1}\left(\phi_{1}(r)+\psi_{1}(r)\right)$ is nondecreasing for large $r$; 
(H5) there exists a positive constant $\varepsilon$ such that

$$
\int_{0}^{\infty} t^{\frac{1+\varepsilon}{m-1}}\left(\phi_{1}(t)+\psi_{1}(t)\right)^{\frac{1}{m-1}} d t<\infty
$$

and

$$
\int_{0}^{\infty} t^{\frac{1+\varepsilon}{l-1}}\left(\phi_{1}(t)+\psi_{1}(t)\right)^{\frac{1}{l-1}} d t<\infty
$$

Our main results are as the following:

Theorem 1. Under the hypotheses (H1)-(H5), equation (1.1) has a positive entire bounded solution $(u, v)$.

From the above theorem, we get the following corollary

Corollary 1. Suppose that $p$ and $q$ are spherically symmetric(i.e. $p(x)=p(|x|, q(x)=q(|x|))$. Under hypotheses (H1)-(H3), (1.1) has one positive solution $(u, v)$.

Suppose further that $\mathrm{P}(\infty)=\mathrm{Q}(\infty)=\infty$, where

$$
\begin{aligned}
& P(\infty):=\lim _{r \rightarrow \infty} P(r), P(r):=\int_{0}^{r}\left(t^{1-N} \int_{0}^{t} s^{N-1} p(s) d s\right)^{\frac{1}{m-1}} d t, r \geq 0 \\
& Q(\infty):=\lim _{r \rightarrow \infty} Q(r), Q(r):=\int_{0}^{r}\left(t^{1-N} \int_{0}^{t} s^{N-1} q(s) d s\right)^{\frac{1}{l-1}} d t, r \geq 0
\end{aligned}
$$

Then every positive radial entire solution $(u, v)$ of $(1.1)$ is large and satisfies

$$
u(r) \geq u(0)+f(v(0)) P(r), v(r) \geq v(0)+g(u(0)) Q(r) . \quad \forall r \geq 0
$$

Corollary 2. Under the assumption (H1)-(H4), if (1.1) has a non-negative radial entire large solution, then at least one of the following two equations hold:

$$
\begin{aligned}
& \int_{0}^{\infty} r^{\frac{1+\varepsilon}{m-1}}(p(r)+q(r))^{\frac{1}{m-1}} d r=\infty, \quad \forall \varepsilon>0 . \\
& \int_{0}^{\infty} r^{\frac{1+\varepsilon}{l-1}}(p(r)+q(r))^{\frac{1}{l-1}} d r=\infty, \quad \forall \varepsilon>0 .
\end{aligned}
$$

Remark 1. By (H1) and (H3), we have

$$
\int_{a}^{\infty} \frac{d s}{\sqrt[d]{F(s)}}=\int_{a}^{\infty} \frac{d s}{\sqrt[d]{G(s)}}=\infty
$$

Remark 2. When $2 \leq d<\infty, \int_{0}^{\infty} r^{\frac{1}{d-1}}(p(x)+q(x))^{\frac{1}{d-1}} d r=\infty$ implies

$$
\int_{0}^{\infty}\left(t^{1-N} \int_{0}^{t} s^{N-1}(p(x)+q(x))(s) d s\right)^{\frac{1}{d-1}} d t=\infty
$$


Remark 3. If $\int_{a}^{\infty} \frac{d s}{\sqrt[d]{f(s)}}<\infty, a>0$, then $\int_{a}^{\infty} \frac{d t}{(f(t))^{d-1}}<\infty$. In other words, if $\int_{a}^{\infty} \frac{d t}{(f(t)) \frac{1}{d-1}}=$ $\infty$, then $\int_{a}^{\infty} \frac{d s}{\sqrt[d]{\mathrm{F}(s)}}=\infty$

Proof. We only need to prove

$$
\frac{(f(t))^{\frac{1}{d-1}}}{s}>\delta^{d}
$$

for $\forall \delta>0$. Then $F(s) \equiv \int_{0}^{s} f(t) d t \leq s f(s) \leq \frac{f d-T(s)}{\delta^{d}}$, and $(F(s))^{-\frac{1}{d}} \geq \frac{\delta}{(f(s)) \frac{1}{d-1}}$. We suppose that (1.6) is not true, then $\exists$ an increasing sequence $\left\{s_{j}\right\}, \lim _{j \rightarrow \infty} s_{j}=\infty$ such that $\frac{\left(f\left(s_{j}\right)\right) \frac{1}{d-1}}{s_{j}}<\frac{1}{j}$, which equals to $f\left(s_{j}\right) \leq\left(\frac{s_{j}}{j}\right)^{d-1}$, then $\left(f\left(s_{j}\right)\right)^{-\frac{1}{d}} \geq\left(\frac{s_{j}}{j}\right)^{-\frac{d-1}{d}}$. Since $f$ is nondecreasing, we get $f(s) \leq f\left(s_{j}\right)$ for all $s \in\left[0, s_{j}\right]$, so $F(s) \leq s f(s) \leq \operatorname{sf}\left(s_{j}\right)$ for all $s \in\left[0, s_{j}\right]$, and

$$
\begin{aligned}
& \int_{s_{1}}^{s_{j}}(F(s))^{-\frac{1}{d}} d s \geq \int_{s_{1}}^{s_{j}}\left(s f\left(s_{j}\right)\right)^{-\frac{1}{d}} d s \\
& \geq\left(\frac{s_{j}}{j}\right)^{-\frac{d-1}{d}} \int_{s_{1}}^{s_{j}} s^{-\frac{1}{d}} d s=j^{1-\frac{1}{d}}\left(1-\left(\frac{s_{1}}{s_{j}}\right)^{1-\frac{1}{d}}\right) \rightarrow \infty
\end{aligned}
$$

This is a contradiction.

In order to prove the Theorem 1, we give the following lemma.

Lemma 1. For any nonnegative $a$ and $b$, we have

$$
\begin{gathered}
(a+b)^{\alpha} \leq a^{\alpha}+b^{\alpha}, \quad \alpha \in(0,1] \\
(a+b)^{\beta} \leq 2^{\beta-1}\left(a^{\beta}+b^{\beta}\right), \quad \beta \in[1, \infty)
\end{gathered}
$$

Proof of Theorem 1. First, we have to find a pair of super-solution, $(\bar{u}, \bar{v})$ and sub-solution, $(\underline{u}, \underline{v})$, which satisfy $\underline{u} \leq \bar{u}$ and $\underline{v} \leq \bar{v}$. Consider the following system of integral equation:

$$
\begin{aligned}
& \underline{u}(r)=\beta+\int_{0}^{r}\left(t^{1-N} \int_{0}^{t} s^{N-1} \phi_{1}(s) f(\underline{v}(s)) d s\right)^{\frac{1}{m-1}} d t, r \geq 0 \\
& \underline{v}(r)=\beta+\int_{0}^{r}\left(t^{1-N} \int_{0}^{t} s^{N-1} \psi_{1}(s) g(\underline{u}(s)) d s\right)^{\frac{1}{l-1}} d t, r \geq 0
\end{aligned}
$$

where $\beta \geq c>0, c$ is in (H3). Let $\left\{\underline{v}_{k}\right\}_{k \geq 0}$ and $\left\{\underline{u}_{k}\right\}_{k \geq 1}$ be the sequence of positive continuous functions defined on $[0, \infty)$ by $\underline{v}_{0}=\beta$,

$$
\begin{aligned}
& \underline{u}_{k}(r)=\beta+\int_{0}^{r}\left(t^{1-N} \int_{0}^{t} s^{N-1} \phi_{1}(s) f\left(\underline{v}_{k-1}(s)\right) d s\right)^{\frac{1}{m-1}} d t, r \geq 0 \\
& \underline{v}_{k}(r)=\beta+\int_{0}^{r}\left(t^{1-N} \int_{0}^{t} s^{N-1} \psi_{1}(s) g\left(\underline{u}_{k-1}(s)\right) d s\right)^{\frac{1}{l-1}} d t, r \geq 0
\end{aligned}
$$


Then, $\underline{v}_{0} \leq \underline{v}_{1}, \underline{\mathfrak{u}}_{k}(r) \geq \beta$, and $\underline{v}_{k}(r) \geq \beta$ for all $r \geq 0, k \in N$. Using the non-decreasing property of $f$ and $g$, we get $\underline{u}_{1}(r) \leq \underline{u}_{2}(r)$ for all $r \geq 0$, then $\underline{v}_{1}(r) \leq \underline{v}_{2}(r)$ for all $r \geq 0$. Continuing this line, we obtain that the sequence $\left\{\underline{\underline{u}}_{k}\right\}$ and $\left\{\underline{v}_{k}\right\}$ are increasing with respect to $k$ for $r \in[0, \infty)$. Besides,

$$
\begin{aligned}
& \underline{u}_{k}^{\prime}(r)=\left(r^{1-N} \int_{0}^{r} s^{N-1} \phi_{1}(s) f\left(\underline{v}_{k-1}(s)\right) d s\right)^{\frac{1}{m-1}} \geq 0, \\
& \underline{u}_{k}^{\prime}(r)=\left(r^{1-N} \int_{0}^{r} s^{N-1} \psi_{1}(s) g\left(\underline{u}_{k-1}(s)\right) d s\right)^{\frac{1}{l-1}} \geq 0,
\end{aligned}
$$

for each $r>0$, and

$$
\left(r^{N-1}\left|\underline{u}_{k}^{\prime}\right|^{m-2} \underline{u}_{k}^{\prime}\right)^{\prime}=r^{N-1} \phi_{1}(r) f\left(\underline{v}_{k-1}(r)\right) \leq r^{N-1} \phi_{1}(r) f\left(\underline{v}_{k}(r)\right)
$$

let

$$
\Theta(r)=\max _{0 \leq t \leq r}\left(\phi_{1}(t)+\psi_{1}(t)\right),
$$

using this and the fact that $\underline{\mathfrak{u}}_{k}^{\prime} \geq 0$, we note that (3) yields

$$
\left(\left(\underline{u}_{k}^{\prime}(r)\right)^{m-1}\right)^{\prime} \leq \Theta(r) f\left(\underline{v}_{k}(r)\right),
$$

Multiply this by $\underline{\mathfrak{u}}_{k}^{\prime}$ and integrate to get

$$
\left(\underline{u}_{k}^{\prime}(r)\right)^{m} \leq \frac{m}{m-1} \Theta(r) \int_{2 \beta}^{v_{k}(r)+u_{k}(r)} f(s) d s
$$

In the same way,

$$
\left(\underline{v}_{k}^{\prime}(r)\right)^{l} \leq \frac{l}{l-1} \Theta(r) \int_{2 \beta}^{v_{k}(r)+u_{k}(r)} g(s) d s
$$

Then from the inequality $\left(\underline{u}_{k}^{\prime}+\underline{v}_{k}^{\prime}\right)^{\mathrm{d}} \leq 2^{\mathrm{d}-1}\left(\left(\underline{u}_{k}^{\prime}\right)^{\mathrm{d}}+\left(\underline{v}_{k}^{\prime}\right)^{\mathrm{d}}\right)$, where $\mathrm{d}=\min \{\mathrm{m}, l\}$, and the above two inequalities, we get

$$
\begin{aligned}
\left(\underline{u}_{k}^{\prime}+\underline{v}_{k}^{\prime}\right)^{\mathrm{d}} & \leq 2^{\mathrm{d}-1}\left(\left(\underline{u}_{k}^{\prime}\right)^{\mathrm{d}}+\left(\underline{v}_{k}^{\prime}\right)^{\mathrm{d}}\right) \\
& \leq 2^{\mathrm{d}-1}\left(\left(\underline{u}_{k}^{\prime}\right)^{\mathrm{m}}+\left(\underline{v}_{k}^{\prime}\right)^{\mathrm{l}}+1\right) \\
& \leq 2^{\mathrm{d}-1}\left(\frac{\mathrm{d}}{\mathrm{d}-1} \Theta(r) \int_{2 \beta}^{v_{k}(r)+u_{k}(r)}(f(s)+g(s)) \mathrm{d} s+1\right) \\
& \leq 2^{\mathrm{d}-1}\left(\frac{\mathrm{d}}{\mathrm{d}-1} \Theta(\mathrm{r})(\mathrm{F}(\underline{\mathrm{u}}+\underline{v})+\mathrm{G}(\underline{\mathrm{u}}+\underline{v}))+1\right)
\end{aligned}
$$

which yields

$$
\begin{aligned}
\underline{u}_{k}^{\prime}+\underline{v}_{k}^{\prime} & \leq 2^{\frac{d-1}{d}}\left(\frac{d}{d-1} \Theta(r)\left(F\left(\underline{u}_{k}+\underline{v}_{k}\right)+G\left(\underline{u}_{k}+\underline{v}_{k}\right)\right)+1\right)^{\frac{1}{d}} \\
& \leq \sqrt[d]{\frac{2^{d-1} d}{d-1} \Theta(r)}\left(F\left(\underline{u}_{k}+\underline{v}_{k}\right)+G\left(\underline{u}_{k}+\underline{v}_{k}\right)\right)^{\frac{1}{d}}+2^{\frac{d-1}{d}}
\end{aligned}
$$


Integrating the above inequality, we get

$$
\begin{aligned}
& \int_{0}^{r} \frac{\underline{u}_{k}^{\prime}(t)+\underline{v}_{k}^{\prime}(t)}{\left(F\left(\underline{u}_{k}(t)+\underline{v}_{k}(t)\right)+G\left(\underline{u}_{k}(t)+\underline{v}_{k}(t)\right)\right)^{\frac{1}{d}}} d t \\
& =\int_{2 \beta}^{v_{k}(r)+u_{k}(r)} \frac{d \tau}{\sqrt[d]{F(\tau)+G(\tau)}} \\
& \leq \int_{0}^{r}\left(\sqrt[d]{\frac{2^{d-1} d \Theta(t)}{d-1}}+C\right) d t
\end{aligned}
$$

where $C=\frac{2^{\frac{d-1}{d}}}{F(2 \beta)+G(2 \beta)}$. We can easily get

$$
H\left(\underline{u}_{k}(r)+\underline{v}_{k}(r)\right) \leq H(2 \beta)+\int_{0}^{r}\left(\sqrt[d]{\frac{2^{d-1} d \Theta(t)}{d-1}}+C\right) d t
$$

As we know that $\mathrm{H}^{-1}$ is increasing on $[0, \infty)$, so

$$
\mathrm{u}_{\mathrm{k}}(\mathrm{r})+v_{\mathrm{k}}(\mathrm{r}) \leq \mathrm{H}^{-1}\left(\mathrm{H}(2 \beta)+\int_{0}^{\mathrm{r}} \sqrt[\mathrm{d}]{\frac{2^{\mathrm{d}-1} \mathrm{~d} \Theta(\mathrm{t})}{\mathrm{d}-1}}+\mathrm{C}\right) \mathrm{dt}, \quad \forall \mathrm{r} \geq 0
$$

Following by the definition of $\underline{\underline{u}}_{k}(r)$ and $\underline{v}_{k}(r)$ and $(H 3)$, we get that the sequence $\left\{\underline{u}_{k}\right\}$ and $\left\{\underline{v}_{k}\right\}$ are bounded and equi-continuous on $\left[0, C_{0}\right]$ for arbitrary $C_{0}>0$. By Arzela-Ascoli theorem, $\left\{\underline{u}_{k}\right\}$ and $\left\{\underline{v}_{k}\right\}$ have subsequence converging uniformly to $\underline{u}$ and $\underline{v}$ on $\left[0, C_{0}\right]$. By the arbitrariness of $C_{0}>0$,we see that $(\underline{u}, \underline{v})$ is a positive entire solution of

$$
\begin{array}{ll}
\Delta_{\mathrm{m}} \underline{\mathrm{u}}=\phi_{1}(\mathrm{r}) \mathrm{f}(\underline{\mathrm{v}}) \geq \mathrm{p}(x) \mathrm{f}(\underline{\mathrm{v}}), \quad x \in \mathbf{R}^{\mathrm{N}} \\
\Delta_{\mathrm{l} \underline{\mathrm{v}}}=\psi_{1}(\mathrm{r}) \mathrm{g}(\underline{\mathrm{u}}) \geq \mathrm{q}(x) \mathrm{g}(\underline{\mathrm{v}}), \quad x \in \mathbf{R}^{\mathrm{N}}
\end{array}
$$

Then, we take conclusion that $(\underline{u}, \underline{v})$ is a positive entire sub-solution of (1.1).

In order to prove $(\underline{u}, \underline{v})$ is bounded, choosing $R>0$, so that $r^{d(N-1)}\left(\phi_{1}(r)+\psi_{1}(r)\right)$ is nondecreasing on $[R, \infty)$ and $\underline{u}(r)>0, \underline{v}(r)>0$. This is possible because of $(H 4)$. Since $(\underline{u}, \underline{v})$ satisfies

$$
\begin{aligned}
& \left(r^{N-1}\left(\underline{u}^{\prime}\right)^{m-1}\right)^{\prime}=r^{N-1} \phi_{1}(r) f(\underline{v}(r)), \\
& \left(r^{N-1}\left(\underline{v}^{\prime}\right)^{l-1}\right)^{\prime}=r^{N-1} \psi_{1}(r) g(\underline{u}(r)) .
\end{aligned}
$$

$\underline{u}^{\prime}(r) \geq 0$ and $\underline{v}^{\prime}(r) \geq 0$ for $r \geq 0$, and (H2) hold, multiplying (8) and (9) by $\underline{u}^{\prime}$ and $\underline{v}^{\prime}$, respectively, and integrating from $\mathrm{R}$ to $\mathrm{r}$. Take (8) as an example,

$$
\int_{R}^{r}\left(s^{N-1}\left(\underline{u}^{\prime}\right)^{m-1}\right)^{\prime} \underline{u}^{\prime}(s) d s=\int_{R}^{r} s^{N-1} \phi_{1}(s) f(\underline{v}(s)) \underline{u}^{\prime}(s) d s,
$$

which implies that

$$
\frac{m-1}{m} r^{N-1}\left(\underline{u}^{\prime}(r)\right)^{m}-\frac{m-1}{m} R^{N-1}\left(\underline{u}^{\prime}(R)\right)^{m}+\frac{N-1}{m} \int_{R}^{r} s^{N-2}\left(\underline{u}^{\prime}(s)\right)^{m} d s=\int_{R}^{r} s^{N-1} \phi_{1}(s) f(\underline{v}(s)) \underline{u}^{\prime}(s) d s
$$


It follows that

$$
r^{N-1}\left(\underline{u}^{\prime}(r)\right)^{m} \leq R^{N-1}\left(\underline{u}^{\prime}(R)\right)^{m}+\frac{m}{m-1} \int_{R}^{r} s^{N-1} \phi_{1}(s) f(\underline{v}(s)) \underline{u}^{\prime}(s) d s
$$

Using the monotonicity of $\mathrm{t}^{\mathrm{N}-1}\left(\phi_{1}(\mathrm{t})+\psi_{1}(\mathrm{t})\right)$ for $\mathrm{t} \geq 0$, we get

$$
r^{N-1}\left(\underline{u}^{\prime}(r)\right)^{m} \leq \bar{C}+\frac{m}{m-1} r^{N-1}\left(\phi_{1}(r)+\psi_{1}(r)\right)(F(\underline{u}(r)+\underline{v}(r))+G(\underline{u}(r)+\underline{v}(r)))
$$

for $r>R$, where $\bar{C}=R^{N-1}\left(\underline{u}^{\prime}(R)\right)^{m}+R^{N-1}\left(\underline{v}^{\prime}(R)\right)^{l}$.

which yields

$$
\underline{u}^{\prime}(r) \leq \sqrt[m]{\bar{C}} r^{\frac{1-N}{m}}+\sqrt[m]{\frac{m}{m-1}\left(\phi_{1}(r)+\psi_{1}(r)\right)}(F(\underline{u}+\underline{v})+G(\underline{u}+\underline{v}))^{\frac{1}{m}}
$$

So

$$
\begin{aligned}
& \underline{u}^{\prime}(r)+\underline{v}^{\prime}(r) \leq C_{1}\left(r^{\frac{1-N}{m}}+r^{\frac{1-N}{l}}\right) \\
& +\left(\sqrt[m]{\frac{m}{m-1}\left(\phi_{1}(r)+\psi_{1}(r)\right)}+\sqrt[l]{\frac{l}{l-1}\left(\phi_{1}(r)+\psi_{1}(r)\right)}\right)\left(2(F(\underline{u}+\underline{v})+G(\underline{u}+\underline{v}))^{\frac{1}{d}}+1\right)
\end{aligned}
$$

and

$$
\begin{aligned}
& \frac{\mathrm{d}}{\mathrm{d} r} \int_{\underline{\mathrm{u}}(R)+\underline{v}(R)}^{\underline{\mathrm{u}}(\mathrm{r})+\underline{\underline{v}}(\mathrm{r})} \frac{\mathrm{d} \tau}{\sqrt[\mathrm{d}]{\mathrm{F}(\tau)+\mathrm{G}(\tau)}} \\
& \leq C_{1}\left(r^{\frac{1-\mathrm{N}}{\mathrm{m}}}+\mathrm{r}^{\frac{1-\mathrm{N}}{\mathrm{l}}}\right)(\mathrm{F}(\underline{\mathrm{u}}+\underline{v})+\mathrm{G}(\underline{\mathrm{u}}+\underline{v}))^{-\frac{1}{\mathrm{~d}}}+\mathrm{h}(\mathrm{r})\left(2+(\mathrm{F}(\underline{\mathrm{u}}+\underline{v})+\mathrm{G}(\underline{\mathrm{u}}+\underline{v}))^{-\frac{1}{\mathrm{~d}}}\right)
\end{aligned}
$$

where $h(r)=\sqrt[m]{\frac{m}{m-1}\left(\phi_{1}(r)+\psi_{1}(r)\right)}+\sqrt[l]{\frac{l}{l-1}\left(\phi_{1}(r)+\psi_{1}(r)\right)}$.

We notice the fact that

$$
F(\underline{u}(r)+\underline{v}(r))+G(\underline{u}(r)+\underline{v}(r)) \geq F(\underline{u}(R)+\underline{v}(R))+G(\underline{u}(R)+\underline{v}(R))=C_{2}
$$

for all $r \geq R$, and

$$
\sqrt[m]{\frac{m}{m-1}\left(\phi_{1}(r)+\psi_{1}(r)\right)} \leq \frac{m}{m-1} \sqrt[m]{r^{1+\varepsilon}\left(\phi_{1}(r)+\psi_{1}(r)\right) r^{-1-\varepsilon}}
$$

Using Young's inequality, we get

$$
\sqrt[m]{\frac{m}{m-1}\left(\phi_{1}(r)+\psi_{1}(r)\right)} \leq \frac{1}{m-1} r^{-1-\varepsilon}+r^{\frac{1+\varepsilon}{m-1}}\left(\phi_{1}(r)+\psi_{1}(r)\right)^{\frac{1}{m-1}} \quad \text { for } \varepsilon>0 .
$$

In the same way,

$$
\left.\sqrt[l]{\frac{l}{l-1}\left(\phi_{1}(r)+\psi_{1}(r)\right)} \leq \frac{1}{l-1} r^{-1-\varepsilon}+r^{\frac{1+\varepsilon}{l-1}}\left(\phi_{1}(r)+\psi_{1}(r)\right)\right)^{\frac{1}{l-1}} \text { for } \varepsilon>0 .
$$


Then integrate (10) from $\mathrm{R}$ to $\mathrm{r}, \mathrm{r} \geq \mathrm{R}$,

$$
\begin{aligned}
& H(\underline{u}(r)+\underline{v}(r))-H(\underline{u}(R)+\underline{v}(R)) \\
& \leq C_{3}+C_{4}\left(\left(\frac{1}{m-1}+\frac{1}{l-1}\right) \frac{R^{-\varepsilon}}{\varepsilon}+\int_{R}^{r} t^{\frac{1+\varepsilon}{m-1}}\left(\phi_{1}+\psi_{1}\right)^{\frac{1}{m-1}} d t+\int_{R}^{r} t^{\frac{1+\varepsilon}{l-1}}\left(\phi_{1}+\psi_{1}\right)^{\frac{1}{l-1}} d t\right)
\end{aligned}
$$

where $C_{3}=\sqrt[d]{C_{2}} C_{1}\left(\frac{m R^{\frac{1+m-N}{m}}}{N-m-1}+\frac{l R^{\frac{1+l-N}{l}}}{N-l-1}\right), C_{4}=2+(F(2 R)+G(2 R))^{-\frac{1}{d}}$.

From (H5), we know

$$
\int_{R}^{r} t^{\frac{1+\varepsilon}{m-1}}\left(\phi_{1}+\psi_{1}\right)^{\frac{1}{m-1}} d t<\infty
$$

and

$$
\int_{R}^{r} t^{\frac{1+\varepsilon}{l-1}}\left(\phi_{1}+\psi_{1}\right)^{\frac{1}{l-1}} d t<\infty
$$

So

$$
\mathrm{H}(\underline{u}(r)+\underline{v})(r)<\infty
$$

Letting $r \rightarrow \infty$, since $\mathrm{H}$ satisfies (H3), we find that $(\underline{u}, \underline{v})$ is bounded .

By now, we have find a pair of bounded sub-solution to (1.1). We still have to find $(\bar{u}, \bar{v})$, which is a bounded super-solution of (1.1), and $\underline{u}(r) \leq \bar{u}(r), \underline{v}(r) \leq \bar{v}(r)$ for all $r \geq 0$. Actually, since $(\underline{u}, \underline{v})$ is nondecreasing and bounded, we have

$$
\lim _{r \rightarrow \infty} \underline{u}(r)=M_{1}>0, \quad \lim _{r \rightarrow \infty} \underline{v}(r)=M_{2}>0 .
$$

Let $\bar{u}(0)=\bar{v}(0)=\max \left\{M_{1}, M_{2}\right\}, \bar{u}^{\prime}(0)=\bar{v}^{\prime}(0)=0$, then, the following system

$$
\begin{array}{ll}
\Delta_{\mathrm{m}} \bar{u}(x)=\phi_{2}(r) f(\bar{v}(r)) & r>0 \\
\Delta_{l} \bar{v}(x)=\psi_{2}(r) g(\bar{u}(r)) & r>0
\end{array}
$$

has a bounded solution $(\bar{u}, \bar{v})$ by the same argument, and it is a supersolution for (1.1). From the above process, we get conclusion that

$$
\underline{\mathrm{u}}(\mathrm{r}) \leq \mathrm{M}_{1} \leq \overline{\mathrm{u}}(\mathrm{r}), \quad \underline{v}(\mathrm{r}) \leq M_{2} \leq \bar{v}(\mathrm{r}) . \quad \forall \mathrm{r} \geq 0 .
$$

The standard super-sub solution principle $[18,20]$ implies that (1.1) has a bounded solution $(u, v)$ satisfying $\underline{u}(x) \leq u(x) \leq \bar{u}$ and $\underline{v}(x) \leq v(x) \leq \bar{v}$ on $\mathbf{R}^{N}$, which is the desired solution. This completes the proof.

\section{Conclusion}

The boundary value quasilinear differential equation systems (1.1) are mathematical models occurring in the studies of the m-Laplace equation, generalized reaction-diffusion theory, nonNewtonian fluid theory, and the turbulent flow of a gas in porous medium. When $m \neq 2$, the 
problem becomes more complicated since certain nice properties in herent to the case $\mathrm{m}=2$ seem to be lost or at least difficult to verify. The main differences between $m=2$ and $m \neq 2$ can be founded in $[8,9]$. When $m=2$, it is well known that all the positive solutions in $C^{2}\left(B_{R}\right)$ of the problem

$$
\left\{\begin{array}{l}
\Delta u+f(u)=0 \text { in } B_{R} \\
u(x)=0 \text { on } \partial B_{R}
\end{array}\right.
$$

are radially symmetric solutions for very general $f($ see $[7])$. Unfortunately, this result does not apply to the case $m \neq 2$. Kichenassary and Smoller showed that there exist many positive nonradial solutions of the above problem for some $f($ see [11]). The major stumbling block in the case of $m \neq 2$ is that certain nice features inherent to the case $m=2$ seem to be lost or at least difficult to verify. In this paper, we first give some necessary preliminary knowledge. Secondly, we further study the existence of positive solutions to problem (1.1) which the right hand side functions are more general based on the method of sub-supersolution.

Received: October 2012. Revised: March 2013.

\section{References.}

[1] F. Cirstea, V. D. Radulescu, Entire solutions blowing up at infinity for semilinear elliptic systems, J. Math. Pures Appl. 81 (2002), 827-846.

[2] Ph. Clement, D.G. de Figueiredo and E. Mitidieri, Positive solutions of semilinear elliptic systems, Comm. in P.D.E. (5/6)17(1992), 923-940.

[3] Ph. Clement, R. Manasevich and E. Mitidieri, Positive solutions for a quasilinear system via blow up, Comm. in Partial Diff. Eqns., (12)18(1993), 2071-2106.

[4] P. L. Felmer, R. Manasevich and F. de Thelin, Existence and uniqueness of positive solutions for certain quasilinear elliptic system, Comm.in P.D.E. 17 (1992), 2013-2029.

[5] J. García-Melián, A remark on uniqueness of large solutions for elliptic systems of competitive type, J.Math.Anal.Appl. 331(2007), 608-616.

[6] A. Ghanmi, H. Mâagli, V.Rădulescu and N.Zeddini, Large and bounded solutions for a class of nonlinear Schrödinger stationary systems, Analysis and Applications 4(2009), 1-14.

[7] B. Gidas, W.M.Ni and L.Nirenberg, Symmetry and related properties via the maximum principle, Comm. Math. Phys. 68(1979), 209-243.

[8] Zongming Guo, Some existence and multiplicity results for a class of quasilinear elliptic eigenvalue problems, Nonlinear Anal. 18(1992), 957-971.

[9] Zongming Guo and J.R.L.Webb, Uniqueness of positive solutions for quasilinear elliptic equations when a parameter is large, Proc.Roy.Soc. Edinburgh, 124A(1994), 189-198. 
[10] Zongming Guo, Existence of positive radial solutions for a class of quasilinear elliptic systems in annular domains, Chinese Journal of Contemporary Math. 17(4) (1996), 337-350.

[11] S. Kichenassamy and J. Smoller, On the existence of radial solutions of quasilinear elliptic equations, Nonlinearity 3(1990), 677-694.

[12] A. V. Lair and A. W. Wood, Existence of entire large positive solutions of semilinear elliptic systems, J. Differential Eqns (2)164 (2000), 380-394.

[13] E. Mitidieri, Nonexistence of positive solutions of semilinear elliptic system in $\mathbf{R}^{\mathbf{N}}$, Diff. Integral Equations 9(1996), 465-479.

[14] E. Mitidieri, A Rellich type identity and applications, Comm. in Partial Diff. Equations 18(1993), 125-171.

[15] E. Mitidieri, G. Sweers and R. van der Vorst, Nonexistence theorems for systems of quasilinear partial differential equations, Differential Integral Equations 8(1995), 1331-1354.

[16] L. A. Peletier and R. van der Vorst, Existence and non-existence of positive solutions of non-linear elliptic systems and the biharmonic equations, Diff. Integral Eqns. 54(1992), 747-767.

[17] X.Wang, A.W.Wood, Existence and nonexistence of entire positive solutions of semilinear elliptic system, J.Math.Anal.Appl. 267(2002), 361-362.

[18] Zuodong Yang, Existence of positive bounded entire solutions for quasilinear elliptic equation, Applied Mathematics and Computation 156(2004), 743-754.

[19] Zuodong Yang, Existence of entire explosive positive radial solutions for a class of quasilinear elliptic systems, J. Math. Anal Appl. 288(2003), 768-783.

[20] Zuodong Yang, Existence of explosive positive solutions of quasilinear elliptic equations, Applied Mathematics and Computation 177(2006), 581-588.

[21] Zuodong Yang and Q. S. Lu, Non-existence of positive radial solutions for a class of quasilinear elliptic system, Comm. Nonlinear Sci. Numer. Simul. (4)5(2000), 184-187.

[22] Zuodong Yang and Qishao Lu, Existence of entire explosive positive radial solutions of sublinear elliptic systems, Communication Nonlinear Science Numerical Simulation 6(2)(2001), 88-92.

[23] Zhijun Zhang ,Yongxiu Shi and Yanxing Xue, Existence of entire solutions for semilinear elliptic systems under Keller-Osserman condition, Electronic Journal of Differential Equation 39(2011), 1-9. 